\title{
EVOLUCIÓN DE LAS RELACIONES TRANSFRONTERIZAS EN EL BIDASOA: DE LA CONURBACIÓN A LA COMMUNAUTÉ
}

\author{
J.C. Alberdi Collantes \\ Departamento de Geografía de la Universidad del País Vasco \\ Facultad de Filología y Geografía e Historia \\ juancruz.alberdi@ehu.es
}

\begin{abstract}
Resumen: Con la entrada de España en la Unión Europea y la desaparición de la frontera se intensifican las relaciones internacionales. Pero también vecinos que habían permanecido alejados se acercan unos a otros. Los municipios de Bajo Bidasoa, es decir, Irún, Hondarribia y Hendaia, conforman una conurbación y como tal deciden hacer frente a las necesidades de su población. Para ello crean el Consorcio transfronterizo del Bidasoa el año 1998 pero las dificultades que supone trabajar en dos países diferentes limita su desarrollo. Frente a ello surgen nuevas instituciones, de origen comarcal, que comienzan a superar algunas de las barreras con las que se topaba el Consorcio, hasta el punto de poner la continuidad de esta entidad en entredicho.
\end{abstract}

Palabras clave: conurbación, agencia de desarrollo, País Vasco, colaboración transfronteriza.

\begin{abstract}
With the ingress of Spain in European Community and the end of the border, international relations are developing. On the other side, neighbours who lived separate now are together. The towns of Bidasoa (Irun, Hendaia and Hondarribia), form a conurbation and they decide to look up the future together. They found the International Consortium of Bidasoa in 1998 but the difficulties of working in two different countries restrict its development. Recently some of they are founding new institutions, gathering villages, and they are overcoming some of the difficulties that the Consortium can not overcome. Now the continuity of the Consortium is in doubt while the new institutions are taking more roles.
\end{abstract}

Keywords: conurbation, development agency, Basque Country, international partnership. 
La integración de España en la Comunidad Europea da inicio a una transformación de todas las estructuras, tanto estatales como regionales, que de manera progresiva conduce a otro escenario económico-social. Pero esta reconversión es especialmente intensa en aquellos territorios y localidades que se habían desarrollado en torno a la actividad fronteriza y que ven ahora desaparecer la que había sido el motor principal de su economía.

La necesidad de responder ante la situación originada lleva a las diferentes entidades públicas a poner en marcha programas específicos dirigidos a reorientar la actividad de las zonas limítrofes. Se suceden iniciativas europeas, estatales y regionales pero son las instituciones locales, los ayuntamientos, los que comparten, producto de la proximidad física, una relación diaria que se materializa a ambos lados de la frontera.

En el Bidasoa se observa la necesidad de superar social, cultural y políticamente la frontera. La colaboración transfronteriza debe contribuir al diseño y gestión de la conurbación y desarrollar una estrategia común se considera necesaria. De este modo se da inicio al Plan Estratégico del Bidasoa en 1993, en el que se engloba al municipio de Hendaia, para constituir en 1998 el Consorcio transfronterizo Bidasoa-Txingudi, una entidad surgida con competencias, presupuestos y con el objetivo de responder a las necesidades de la conurbación internacional del Bidasoa.

El Consorcio, década y media después, continúa con la labor de potenciar la relación de las comunidades que viven a ambos lados de la ría tratando de hacer frente al día a día de las necesidades que una conurbación plantea. Su quehacer, sin embargo, es dificultoso y los resultados obtenidos son escasos. Las trabas administrativas que cualquier iniciativa que quiera ponerse en marcha a ambos lados de la frontera tiene que sortear son numerosas. Como consecuencia, muchos de los proyectos iniciados no prosperan por inviabilidad y, a veces, por hastío. Estas dificultades se han visto recientemente incrementadas con el surgimiento de nuevas instituciones que responden a mandatos estatales o regionales y que no tienen en cuenta la peculiaridad de los territorios fronterizos. Como consecuencia se crean nuevas alianzas municipales dejándose de primar las relaciones fronterizas.

A pesar del escaso avance administrativo la conurbación del Bidasoa está viva. La población cambia de residencia, saltándose la barrera fronteriza, la crisis económica potencia la búsqueda de nuevos mercados y las relaciones personales y económicas se incrementan. El Bidasoa es, ahora más que nunca, una unidad física y social.

En este trabajo trataremos de poner en relieve el nuevo escenario en el que se ve inmersa la conurbación, especialmente Hendaia, un municipio en el que algo más de un tercio de la población es originaria del otro lado de la frontera. Al mismo tiempo reflejaremos los logros del Consorcio en sus años de andadura, sus dificultades para avanzar y, en un escenario difícil, sus nuevas alianzas con las realidades administrativas surgidas a raíz de la fundación de las Communauté en Francia, como medio de abordar el desarrollo económico entre los vecinos del Bidasoa. 
El desarrollo de este trabajo lo abordamos recurriendo a dos metodologías prioritarias. En primer lugar, mediante el análisis de la información que las instituciones implicadas van generando tanto de manera escrita como en la red. Especial relevancia tienen los informes anuales de la Agencias de Desarrollo Bidasoa Activa, en los que se incluye la actividad del Consorcio Transfronterizo, el observatorio del proyecto Innov Mugabe, donde se recogen referencias estadísticas actualizadas de los municipios de un lado y otro de la frontera, y la información que la Communaté Sud Pays Basque va publicando en su web. En segundo lugar, el recurso a la entrevista como medio de obtener información y contrastar opiniones y valoraciones. En la realización de este trabajo se entrevistara a los dos técnicos del Consorcio, al responsable de Bidasoa Activa y al técnico que dirige el proyecto Innov Mugabe.

\section{Aproximación a la Conurbación del Bidasoa}

La Conurbación recibe el nombre de Bidasoa-Txingudi en referencia a dos accidentes geográficos fundamentales en su configuración como el es río Bidasoa y la bahía de Txingudi. Este río nace en los montes de Navarra y desemboca en la bahía de Txingudi marcando el límite natural entre España y Francia. Esta constituida por los municipios de Irun y Hondarribia, administrativamente en la Comunidad Autónoma del País Vasco, y el municipio de Hendaia, dependiente del Departamento de los Pirineos Atlánticos.

La función fronteriza que cumple ha afectado en multitud de aspectos de su configuración, desde la fundación de sus villas hasta su organización contemporanea. Irun, desarrollado principalmente sobre los aluviones originados por el río Bidasoa concentra la mayoría de las infraestructuras económicas del área y, junto a ello, continúa incrementando su peso poblacional, con 61.239 habitantes en la actualidad. Principal paso de mercancías rodadas de la Península Ibérica, saca partido de su ubicación estratégica desarrollando todo tipo de servicios dirigidos a compañías de transporte. La especialización de la ciudad se manifiesta en el peso ocupacional de los servicios, que dan empleo al $70.2 \%$ de su población.

Hendaia, con 15.370 habitantes, se caracteriza por la diversidad de funciones que se desarrollan en su término. Entre éstas predominan los servicios, que dan empleo al 79.2\% de su activo, dominando la rama del turismo y del comercio y, en su segundo lugar, el empleo en transporte y comunicaciones. También es destacable su desarrollo industrial, que aporta el 19,7\% del empleo del municipio y que se ubica principalmente en el polígono Joncaux. Junto al transporte cabe destacar la actividad comercial municipal, distribuida entre el pequeño comercio que se concentra en el casco urbano y el comercio al por mayor y centro comerciales, concentrados en el polígono de Joncaux. Esta actividad se ve reforzada en época estival, momento en el cual Hendaia llega a quintuplicar la población residente. Cuenta con 22 hoteles, 7.215 plazas de camping, 270 apartamentos turísticos y alrededor de 5000 residencias secundarias. 


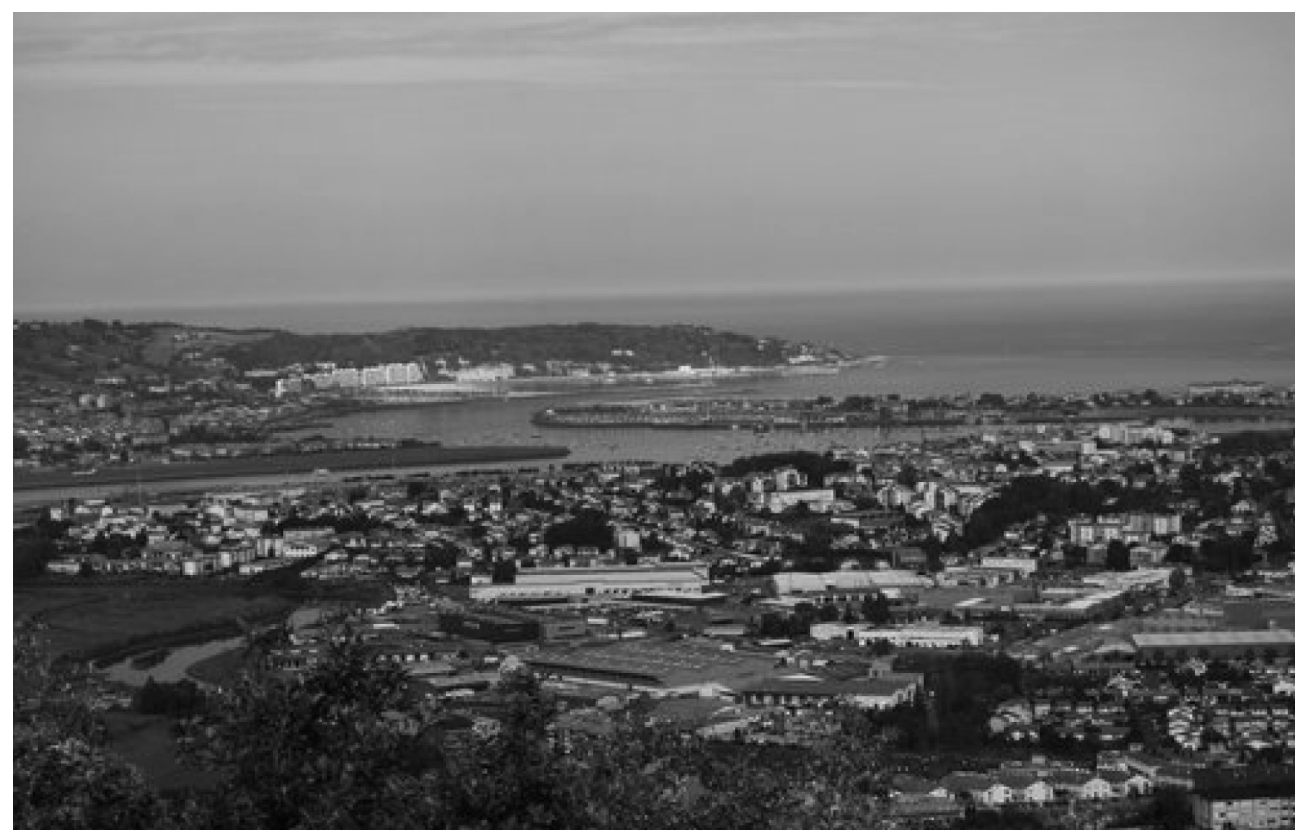

Figura 1. Bahia de Txingudi.

Fuente: www.bidasoa-txingudi.com

Hondarribia, ubicada en la margen izquierda del río Bidasoa, protegida al oeste con las estribaciones del monte Jaizkibel, desarrolla su casco histórico en un pequeño promontorio rodeado de murallas mientras, tras éstas, surge el barrio de la Marina, un poblado típico de pescadores que contrasta con la nobleza del casco medieval. Todo ello lleva a aportar a Hondarribia el título de Monumento Histórico Nacional. En la actualidad el municipio, con 16.500 habitantes, desarrolla su función residencial, atrayendo a población que trabaja en Irun y en San Sebastián mientras las actividades turísticas y recreativas adquieren cada vez más presencia. Como consecuencia, cabe destacar la importancia que adquieren las actividades terciarias, con más del 88 \% de sus habitantes empleado en estas labores.

Pero, además de las peculiaridades propias de cada municipio, uno de los aspectos que caracterizan a la Conurbación y que le aporta el carácter tan especial de su ubicación en una zona limítrofe es, sin duda alguna, el trasvase de población que se está produciendo de un lado a otro de la frontera y que se refleja muy bien en la caracterización actual del municipio de Hendaia.

El movimiento de población desde Gipuzkoa hacia Hendaia y los municipios colindantes no es nuevo, pero tiende a incrementarse. Actualmente, al menos uno de cada 
tres vecinos de la localidad vascofrancesa es de procedencia guipuzcoana. Según Salaberry (2012), alcalde de Hendaia, citando los últimos datos que el consistorio ha recibido del Insee, en torno al 28\% de los 15.370 habitantes actuales es de origen guipuzcoano cuando en 1999, según señala el alcalde, eran el 13\%. Los técnicos del Consorcio, apoyados en estudios de matriculación y en información municipal referente a altas de electricidad e impuestos, según nos señalan en la entrevista, elevan ese porcentaje al $48 \%$ de la población. Y la tendencia no parece que, al menos a corto plazo, vaya a atenuarse puesto que se está acometiendo en este momento la construcción de un nuevo barrio en la ciudad, promovido y dirigido desde y para la población de Gipuzkoa ${ }^{1}$.

Tabla 1. Empleo por sectores económicos \% (2011)

\begin{tabular}{|l|c|c|c|}
\hline & Hendaia & Hondarribia & Irun \\
\hline Empleo sector industrial (\%) & 12,91 & 3,6 & 18,65 \\
Empleo sector construcción (\%) & 4,71 & 6,2 & 16,6 \\
Empleo sector servicios (\%) & 81,37 & 80,57 & 76,09 \\
\hline
\end{tabular}

Fuente: www.bidasoa-subpaysbasque.com

\section{El Consorcio Transfronterizo Bidasoa-Txingudi, un proyecto jurídico común}

La apertura de la frontera y la libre circulación de personas y mercancías constituyen retos y realidades a afrontar por los municipios de la comarca. Ante los retos presentados se ve cada vez más necesario crear una instancia que coordine las estructuras administrativas e institucionales para reforzar la cooperación transfronteriza. Para tal fin se constituye un comité permanente en el que recae la función de realizar el seguimiento de los programas y proyectos en curso.

El primer paso en la materialización del marco jurídico necesario para la colaboración lo constituye el convenio Marco Europeo de Cooperación Transfronteriza de las colectividades o autoridades territoriales, que entró en vigor en 1981. El objeto del convenio

1. En concreto, la promoción Entrepuentes creará en Hendaya 356 nuevas viviendas, con cerca de 2.800 metros cuadrados de locales comerciales, dos aparcamientos públicos con 550 plazas y un hotel de 60 habitaciones. Las viviendas, en las que se prevé vivan cerca de 1.000 nuevos vecinos, se comercializan en la inmobiliaria Arrigain, con sede en el centro de San Sebastián, desde donde señalan que una importantísima mayoría de clientes que está comprando estas casas son guipuzcoanos, con pretensión de establecer su primera vivienda (www.diariovasco.com. 6-2-2012). 
Marco es el de facilitar y promover la cooperación transfronteriza entre colectividades o autoridades territoriales mediante la promoción de la conclusión de acuerdos necesarios pero estableciendo como límite el respeto de las competencias constitucionales de las partes. El convenio marco es objeto de al menos dos protocolos adicionales, en los que se viene a reconocer la posibilidad de crear Organismos de Cooperación Transfronteriza, además del reconocimiento del derecho de las colectividades territoriales de concluir acuerdos con autoridades o colectividades de otros estados.

El tratado de Baiona (1995) constituye el paso realizado por los Estados Español y Francés para facilitar la aplicación del convenio Marco Europeo. Se pretende dotar a las entidades territoriales de un marco jurídico adecuado para llevar a cabo la aplicación de la cooperación transfronteriza. El tratado de Baiona, con su límite de aplicabilidad territorial, establece el contexto legal concreto en el que se basa la experiencia del Consorcio Bidasoa-Txingudi, exigiendo el tratado la conclusión de un convenio entre las entidades territoriales que pretendan crear y gestionar equipamientos o servicios públicos y coordinar sus decisiones en los ámbitos de interés común, que se firma finalmente el 23 de Diciembre de $1998^{2}$. El convenio, en principio, se firma con el objeto de cumplir los fines que de manera resumida a continuación describimos:

- Turismo. Entre otros aspectos se pretende realizar una oferta común de los tres municipios, seguir una política similar, promocionarse de manera conjunta, crear un centro de iniciativas dirigidas desde el mismo Consorcio...

- Cultura: promover el aprendizaje de las diferentes lenguas, realizar cursos de formación, realización de manifestaciones culturales...

- Social: establecimiento de un servicio común de acogida de colectivos inmigrantes con dificultades, puesta en marcha de una oficina de información social, asesoramiento a profesionales y ciudadanos sobre legislación social y sanitaria...

- Desarrollo económico: creación de una célula de información, asesoramiento y de formación para los impulsores de proyectos, organización de transportes regulares fronterizos, favorecer el establecimiento de una plataforma intermodal y participar en su gestión...

Los ayuntamientos de Hendaia, Hondarribia e Irun, por tanto, a fin de impulsar y potenciar la cooperación y la actuación común, crean el Consorcio Transfronterizo Bidasoa-Txingudi como entidad asociativa de entidades locales con personalidad jurídica de carácter público.

\footnotetext{
2. Para profundizar en los antecedentes y objetivos del Consorcio consultar su pagina web: www.bidasoa-txingudi.com
} 
Bidasoa-Txingudi tiene su sede en Irun y, por tanto, el derecho aplicable a las obligaciones de las partes que suscriben el presente convenio es el derecho español. Por consiguiente, las controversias jurídicas que en su desarrollo y aplicación se produzcan se resolverán ante la jurisdicción contencioso-administrativa española.

El reparto financiero de los gastos de funcionamiento tiene lugar conforme a la siguiente distribución: ayuntamiento de Hendaia - 25\%; ayuntamiento de Hondarribia $25 \%$ y ayuntamiento de Irun - 50\%. La incorporación de nuevos miembros al Consorcio no queda cerrada y se apunta que ésta requerirá la aprobación y formalización de un convenio de adhesión en el que se incluirán las modificaciones estatutarias que correspondan. Al mismo tiempo el acuerdo no se plantea de una manera indefinida sino que tiene una duración de diez años desde la fecha de su firma.

Respecto a la figura elegida en la materialización del Consorcio, nace como una administración pública de carácter extraordinario, consideración que se produce por la necesidad que tiene de cumplir tanto la legislación española como la francesa. En principio, se regula en función de la legislación española pero todas sus iniciativas han de estar avaladas por la administración francesa, al ser un ámbito de actuación supranacional. Ello requiere un trabajo de coordinación interadministrativa muy importante y casi imposible una vez que se supera el ámbito competencial municipal.

En relación a su régimen interno, el Consorcio esta gobernado por un Consejo General compuesto por los tres alcaldes junto a dos representantes de cada municipio. En este consejo cada persona tiene un voto, independientemente del presupuesto que aporte cada municipio (Irun el 50\%). Sin embargo, para que un proyecto pueda ser iniciado necesita al menos el apoyo de dos de los representantes de cada villa, lo que en la práctica lleva a asegurar el consenso.

Para la puesta en marcha de acciones de promoción cuenta con la colaboración de Bidasoa Activa, agencia con la que mantiene un convenio de gestión desde la creación del Consorcio y que le aporta todo el apoyo técnico necesario en la materialización de las iniciativas planteadas. En gran medida, aunque con una composición y objetivos diferentes, la coordinación del Consorcio ha pasado a ser una actividad más de Bidasoa Activa, hasta el punto de que es desde las oficinas de esta entidad desde las que se da soporte físico a todas sus labores ${ }^{3}$.

La agencia de Desarrollo del Bidasoa (Bidasoa Activa) se crea en Junio de 1992 a iniciativa de los ayuntamientos de Irun y Hondarribia y con el refrendo del Departa-

3. Si tomamos como referencia el presupuesto que el Consorcio Bidasoa-Txingudi aprueba para el año 2000 observamos cómo de un total de 62 millones de pesetas 45 millones son destinados tienen como beneficiario a la agencia de desarrollo Bidasoa Activa (www.bidasoa-txingudi.com. 2005). 
mento de Economía de la Diputación Foral de Gipuzkoa ${ }^{4}$. Se constituye como una Sociedad Anónima de capital público con el objeto de eliminar las dificultades y trabas administrativas que, en muchos casos, plantea la administración local ${ }^{5}$.

El objeto social con el que se constituye, según su primer presidente, RICO DEL AMO (1994), radica en la promoción, potenciación y desarrollo de los recursos endógenos de la comarca. Estas actuaciones se integran dentro de una nueva concepción de modelos descentralizados de intervención económica en la que son los municipios, partiendo de una dimensión local, de una estructura y un mercado de trabajo diferenciados, los que deberían jugar un papel fundamental.

Fruto de la madurez de la Entidad, Bidasoa Activa y el Consorcio Transfronterizo firman, desde su creación, un convenio de colaboración según el cual la primera asume la función de dinamizar las actividades de la segunda. Bidasoa Activa cede sus instalaciones, contrata el personal que va a dirigir las labores técnicas del Consorcio y gestiona sus proyectos. El Consorcio es una estructura independiente, con unos objetivos, dirección y funcionamiento particular pero el día a día de su actividad ha estado ligada desde su creación a la actividad de la Agencia de Desarrollo.

\section{El Consorcio Transfronterizo, sin embargo, no acaba de desarrollarse}

Una vez firmado el tratado, el Consorcio transfronterizo comienza a poner en marcha diferentes iniciativas encaminadas a alcanzar los objetivos propuestos. En términos generales destacan acciones en el ámbito de la cultura y el deporte, el impulso al proyecto de revalorización del patrimonio de la bahía de Txingudi y el avance en la puesta en marcha de acciones de promoción turística en las tres ciudades.

Profundizando en las peculiaridades de cada campo, se analizan las memorias anuales que publica Bidasoa Activa y que recogen también la actividad del Consorcio, sea

4. Un origen, por otro lado, habitual en zonas con problemas estructurales graves. Por ejemplo, como señala Llusà (2001), la agencia de desarrollo local del Bages Sud también es creada a finales de los ochenta con el apoyo del INEM y de la Diputación de Barcelona, lo que permitió el desarrollo de nuevas políticas de concertación entre el sector público y el privado.

5. Según señala Sese (1994), además de la coyuntura socio-económica de la comarca del Bidasoa otro de los aspectos determinantes para la constitución de Adebisa-Bidasoa Activa fue el crecimiento de diversos servicios y programas en torno al fomento de empleo y la formación iniciados en 1985 por el ayuntamiento de Irun, como era la formación ocupacional, la figura de dinamizador de empleo promovida por el INEM, un servicio de estudios, una oficina dirigida a la información y asesoramiento empresarial o el comité de empleo y formación promovido por el Gobierno Vasco, para los que se crea un departamento específico en el mismo ayuntamiento. Posteriormente se acuerda el ampliar el campo de actuación de este departamento a toda la comarca mediante la constitución de una sociedad instrumental. 
con la colaboración de los técnicos de una u otra entidad. En ellas observamos que ni las acciones emprendidas ni los campos trabajados presentan variaciones importantes en su década y media de andadura.

Analizando las actuaciones del Consorcio en la entrevista con sus técnicos se valora más pormenorizadamente su alcance. Como señalan, en el periodo que comprende los últimos diez años apenas ha abordado nuevos campos pero si ha reforzado el trabajo desarrollado los años anteriores multiplicando el número de iniciativas en ámbitos como el turismo, la cultura, el deporte.... En general, se resalta la posibilidad que una conurbación transfronteriza tiene a la hora de promocionarse en dos regiones y estados diferentes.

En Turismo, se confirma como principal objetivo de trabajo la consolidación del destino Bidasoa como nueva referencia vacacional principalmente en los sectores de los estados francés y español. A dicho efecto, se ha configurado una serie de objetivos que se han venido reforzando a raíz de las experiencias adquiridas desde la colaboración entre las oficinas de turismo comarcales. Éstos consisten en la edición conjunta de herramientas turísticas sobre la bahía, actuaciones promocionales del Destino Bidasoa y la realización de campañas publicitarias para la captación de turistas en temporada baja. También se repercute en el tejido turístico comarcal por lograr asentar de forma definitiva la importancia de la cooperación y la complementariedad turística fomentada por la Comisión de Turismo del Consorcio. Los resultados son innegables y hoy en día el trasiego de turistas de un lado a otro de la frontera se realiza con toda normalidad, obteniendo toda la comarca beneficio de este hecho.

El grupo de trabajo de cultura y deporte es uno de los primeros que empezó a funcionar incluso sin estar el Consorcio constituido como tal, impulsando en 1.997 el primer "día de Txingudi", siendo esta fiesta el inicio de numerosas iniciativas impulsadas desde este grupo. El principal avance se observa en la difusión que tienen ahora estos eventos. Al ser organizados por asociaciones locales que se relacionan en ámbitos regionales y nacionales diferentes, los contactos y el público que cada uno atrae es diverso y, como consecuencia, se enriquece enormemente este tipo de actos.

Fuera de los ámbitos trabajados los resultados son escasos. Inicialmente el Consorcio nace para promocionar áreas de desarrollo económico, a lo largo de su andadura se plantea asumir servicios públicos como la recogida de residuos sólidos o el abastecimiento de agua pero, generalmente por trabas administrativas, nunca llegan a materializarse. Son pocas las acciones iniciadas en estas áreas, con resultados que habitualmente no superan la fase de proyecto y que rápidamente son aparcados ${ }^{6}$.

6. De hecho es el apartado económico donde se observa la principal necesidad de avanzar en la colaboración transfronteriza no sólo municipal sino y especialmente regional. Como señalan Ibarra e Ikardo (1997), una de las principales dificultades la constituyen las diferencias en los marcos jurídico, económico 
De hecho, como señala Salaberry (2012), alcalde de Hendaia y actual presidente del Consorcio, "Lo transfronterizo no ha ido tan rápido como debía y ahora lo estamos pagando". Salaberry muestra la dificultad que tiene el Consorcio para resolver los problemas que día a día tiene el ciudadano que vive en la Conurbación? . Apunta que "En el Consorcio hay un planteamiento común, pero la realidad es que las leyes son diferentes. No son cosas de vida o muerte, pero te queda un sabor amargo cuando intentas resolver un problema transfronterizo y no puedes porque te enredas en cuestiones jurídicas de competencias, si decide Vitoria o Madrid, por ejemplo. Queda mucho por hacer para que ésto vaya tan fluido como debiera".

Las declaraciones del Alcalde de Hendaia muestran la dificultad que tienen los Ayuntamientos para poner en marcha iniciativas que respondan a las necesidades que una conurbación tiene en materia de transporte, servicios públicos, medio ambiente....Mientras tanto esta colaboración se queda, como apunta el regidor, en promover acciones que ayudan a mantener cierta relación social, es decir, actividades culturales y deportivas, campos tradicionalmente trabajados por el Consorcio.

La integración administrativa de la conurbación no avanza. Es más, a pesar del interés de las autoridades, en cierta medida retrocede porque surgen nuevas entidades que dan respuesta a las necesidades de los ciudadanos y que impulsan otra serie de alianzas municipales, independientes de la del Consorcio. Este es el caso de la Communauté de Communes Sud Pays Basque, creada el año 2005 y que va asumiendo competencias financieramente muy pesadas para los ayuntamientos como la recogida de basuras y el abastecimiento de agua, que se planteaban de manera colectiva en la Conurbación, limitándose los campos de trabajo comunitario a un lado y otro de la frontera o, en el mejor de los casos, quedando a expensas de acuerdos ya no entre tres sino entre doce ayuntamientos por un lado y dos por otro.

\section{Nuevas entidades, ¿dificultad u oportunidad?}

La Comunidad de Comunas (Communauté de Communes) es una estructura administrativa de Francia. Se trata de una Entidad pública de cooperación intercomunal (Établissement public de coopération intercommunale) que ejerce, en lugar de las comunas

\footnotetext{
y cultural entre los territorios que podrían colaborar en la realización de infraestructuras. Otro de los escollos sería la funcionalidad de unión de áreas metropolitanas que caracteriza a las infraestructuras de transporte y comunicación.

7. Declaraciones realizadas por Jean-Baptiste Sallaberry, Presidente del Consorcio y alcalde de Hendaia, una vez que accede a este cargo y en las que se muestra la dificultad que atraviesa el Consorcio para hacer frente a las demandas de una conurbación. En el mismo artículo, a pesar de las dificultades, el Alcalde afirma la apuesta que los ayuntamientos hacen por mantener y desarrollar esta entidad. Consular www.diariovasco.com, el 17-11-2012.
} 
miembros, un cierto número de competencias definidas por la ley de 6 de febrero de 1992 relativa a la administración territorial de la República francesa, modificada por la ley de 12 de julio de 1999, y posteriormente por la ley de 27 de febrero de 2002. Las disposiciones correspondientes están codificadas en los textos legislativos y reglamentarios del Código general de las colectividades territoriales (Code général des collectivités territorials, CGCT). Contrariamente al caso de las otras intercommunalités (Communautés d'agglomération y Communautés urbaines), las Communautés de Communes no están sometidas a un mínimo de población. La única constricción es la continuidad geográfica.

Estas Communauté están administradas por un consejo comunitario. Los delegados de cada comuna son elegidos por los consejos municipales, cada comuna tiene al menos un delegado y ninguna puede tener más de la mitad del total de los mismos. Asumen diferentes competencias, entre las obligatorias está el desarrollo económico, la gestión territorial y al menos una a elegir de un bloque de cuatro que engloba al medio natural, a la política de vivienda y asuntos sociales, a la gestión de la red viaria o a las dotaciones culturales, deportivos y de enseñanza infantil y primaria. Aún así, el consejo puede también elegir aquellas competencias facultativas que él mismo decida. Las comunas, por su parte, eligen, dentro de los bloques, las competencias precisas que ellas delegan en la Communauté. A partir del momento en que las competencias son delegadas, las comunas ya no pueden ejercerlas.

La Communauté Sud Pays Basque se crea en el año 2005, englobando a un total de 12 municipios y a una población de 64.863 habitantes, es decir al 20\% de la población residente en el País Vasco Francés. Constituyen un consejo, con electos de todos los municipios implicados, representados en los 37 consejeros. Las decisiones son sometidas a votación y las reuniones del consejo tienen carácter público.

La mitad de la población vive entre Hendaia y San Juan de Luz y otra cuarta parte en la prolongación de estos municipios en Ziburu y Urruña. El resto lo constituyen localidades más discretas, antaño rurales, salpicadas de caseríos dispersos, y actualmente sometidas a los movimientos pendulares de población que trabaja en el medio urbano y que reside en este hábitat disperso ${ }^{8}$.

El área estudiada, la parte sur de la Aglomeración del País Vasco Francés (la aglomeración tiene en Bayona y Biarritz sus principales núcleos de referencia), presenta una fuerte especialización en el sector servicios, que da empleo aproximadamente al 80\% de su población. El turismo es, en gran medida, el motor de su economía e, incluso en mayor medida que Hendaia, en San Juan de Luz, ciudad balneario. El mayor dina-

8. A raíz de la aprobación del proyecto Innov Mugabe Adebisa y la Communauté Sud Pays Basque crear un observatorio en el que se recogen los datos más significativos de la población y de la economía de las localidades que componen esta comarca. Se pueden consultar en la wed del proyecto www.bidasoa-sudpaysbasque.com 
mismo población se ha trasladado de estas poblaciones balneario hacia el interior más próximo. Urruna e incluso antaño localidades rurales como Senpere duplican su población en apenas dos décadas preferentemente por migraciones internas, generalmente población joven que huye de los altos precios de la vivienda en la costa.

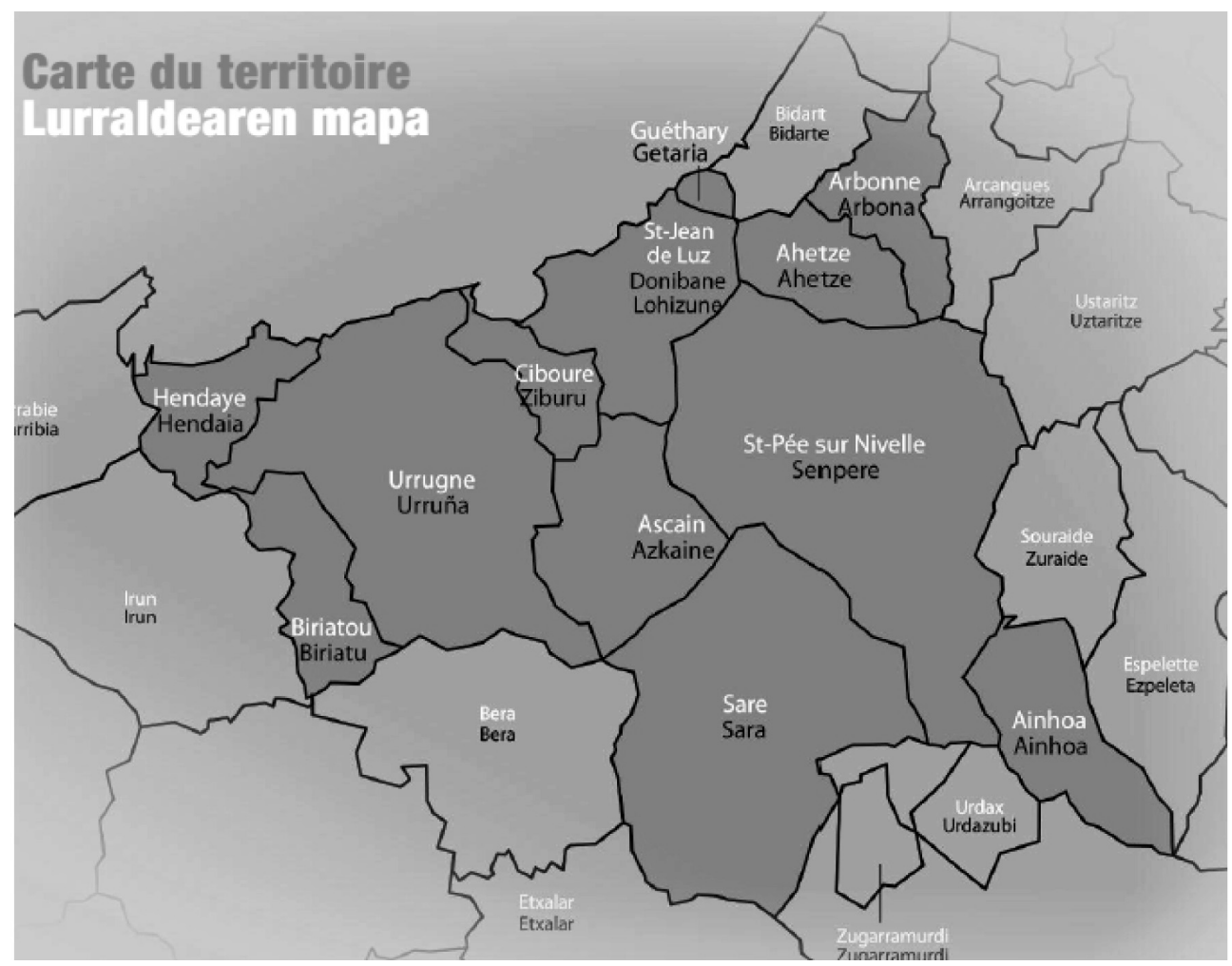

Figura 2. Communauté Sud Pays Basque.

Fuente: www.agglo-sudpaysbasque.fr

La Communauté se dota de una serie de competencias, algunas obligatorias como la ordenación territorial o el desarrollo económico y otras designadas por interés de sus miembros, hasta un total de 11, entre las que se encuentran la de vivienda, infraestructuras, abastecimiento de agua, saneamiento, medio ambiente, cultura y relaciones transnacionales, entre otras.

A partir del año 2013 y tras superar Hendaia los 15000 habitantes, la Communauté adquiere la subcategoría de Aglomeración, aspecto que no cambia su dinámica anterior pero que le va a llevar a adquirir nuevas competencias que aún no había desarro- 
llado, algunas de ellas de manera obligatoria. Mantenimiento de infraestructuras, transporte urbano, desarrollo de espacios públicos... están entre las nuevas competencias que la Aglomeración Sud Pays Basque tiene que comenzar a gestionar?.

Tabla 2. Población y empleo en la Communauté Sud Pays Basque (2011)

\begin{tabular}{|l|r|}
\hline Población total (habitantes) & 63.014 \\
Tasa de paro (\%) & 10,7 \\
Número de desempleados & 2.970 \\
№ empleos & 21.538 \\
Empleo sector industrial (\%) & 11,9 \\
Empleo sector construcción (\%) & 7,6 \\
Empleo sector servicios (\%) & 78,4 \\
\hline
\end{tabular}

Fuente: www.bidasoa-subpaysbasque.com

En los 7 años de andadura de la Communauté vasca los 12 municipios han ido desarrollando acciones diversas en cada una de aquellas competencias de las que se han dotado $^{10}$. En materia de vivienda, por ejemplo, contralan la construcción de nueva vivienda, un total de 4.037 nuevas viviendas en este periodo, además de promover y apoyar el alquiler de vivienda social. En el área de ordenación territorial, la Communauté ha adecuado su normativa territorial de manera conjunta además de abordar la ordenación del transporte público en la comarca. También ha ejecutado infraestructuras comunitarias como la puesta en marcha de una perrera comunal. A su vez, ha asumido las competencias de saneamiento y abastecimiento de agua en su integridad, desde su tarifación y recaudación a su mantenimiento.

La competencia en ordenación territorial le ha dado la potestad de dirigir actuaciones en esta línea. Así ha planificado el desarrollo de polígonos destinados a la promoción de actividades económicas, incluso adquiriendo suelo, todo ello desde una visión global. Pero también ha impulsado la consecución de un semillero de empresas, dando cabida a 20 proyectos empresariales y ha apoyado económicamente a más de 100 proyectos económicos.

9. Con la nueva denominación el link de la Communauté también cambia. En su web se puede consultar multitud de información sobre el funcionamiento, proyectos y competencias de esta aglomeración desde que comenzó a funcionar. www.agglo-sudpaysbasque.fr

10. Para profundizar en las acciones que durante el periorido 2005-2012 ha realizado la Communauté consultar la publicación “2005-2012. D’une Communauté de Comunes à une Agglomération”, recogida en la web de la entidad. Además del informe, en la misma web se recoge de manera individual los objetivos, actuaciones y planes de cada una de las áreas en las que tiene competencias. 
El turismo es otra de las líneas de actuación que se ha decidido emprender de manera conjunta, especialmente la conexión entre el turismo de costa y el de interior. Acciones de promoción, recorridos y apoyo a proyectos empresariales y artesanos están entre las actividades más reseñables. Junto a ello, la promoción de los espacios naturales, de gran importancia en un área que marca el final del Pirineo y que en gran medida está integrada en la red natura 2000, junto a la asunción de las competencias culturales y el Euskera, recurso que completa la oferta de una zona eminentemente turística, son ahora también asumidas por la Communauté.

Además, los ayuntamientos deciden que sea la Communauté la que aborde las relaciones transfronterizas. Aunque sea Hendaia la localidad en la que éstas tienen mayor presencia, son muchos los municipios limítrofes, como Biariatu, Urruña, Sara, Senpere o Ainhoa, marcando buena parte de la muga del Bidasoa, ya con localidades Navarras con Bera o Baztan. Se suceden multitud de proyectos entre localidades de un lado y otro de la frontera, algunos relacionados con saneamiento comunitario, otros con el control de la calidad de las aguas, la promoción de espacios naturales.... Uno de ellos, Innov Mugabe, está dirigido a promocionar la relación entre empresas de ambos lados de la frontera. Tras un acuerdo entre Bidasoa Activa y la Communauté Sud Pays Basque se da inicio a partir del año 2010 a un proyecto de colaboración entre empresas, una iniciativa que trata de romper con el tradicional recelo entre la población de un lado y otro del Bidasoa.

\section{Innov Mugabe: cooperación empresarial transfronteriza}

Innov Mugabe es un proyecto de desarrollo empresarial que, a través de colaboraciones entre empresas, universidades y centros tecnológicos del Bidasoa y del Sud Pays Basque, quiere trabajar e impulsar proyectos conjuntos en torno a materias que promuevan y faciliten procesos de Innovación.

En línea con el programa europea POCTEFA (Programa Operativo de Cooperación Territorial entre Francia, España y Andorra) el proyecto se aborda desde la perspectiva de la mejora competitiva del tejido empresarial a partir del aprovechamiento y la potenciación de las oportunidades derivadas de su condición transfronteriza.

Las acciones van dirigidas a empresas de todos los sectores, situadas principalmente en los polígonos empresariales de las Comarcas del Bidasoa y de Sud Pays Basque. Cuenta con dos socios participantes, Bidasoa Activa y la Communauté de Communes du Sud Pays Basque (a partir del 2013 aglomeración) y dispone de una web oficial del proyecto: www.bidasoa-sudpaysbasque.com. Como tal inicia su andadura en marzo de 2010, con una duración de 28 meses y un presupuesto de $1.195 .000 €$, cofinanciados en un $65 \%$ por el Feder y repartidos más o menos a partes iguales entre sus dos socios. 
Coincidiendo con la firma de acta de constitución del Comité Político de Innov Mugabe, la presidenta de Bidasoa Activa, Goizane Álvarez (2010), resalto que "El proyecto liderado por Bidasoa Activa tiene un doble objetivo. Por un lado, compartir los instrumentos existentes de gestión de la información y del conocimiento del territorio, y por otro lado, crear y consolidar las bases para la cooperación transfronterizo a través de proyectos piloto entre empresas ${ }^{11 "}$.

En este sentido, las primeras acciones que se han llevado a cabo desde el arranque del proyecto incluyen la definición de un plan de comunicación con el diseño de la imagen de marca del proyecto y su aplicación a la difusión de todas las actividades relacionadas con el proyecto, reflejando las ideas de Innovación y cooperación sin fronteras. Las acciones que conforman el proyecto Innov Mugabe son las siguientes:

- Acción 1. Plataforma local de iniciativas fronterizas. Recopilación y puesta en común de datos y experiencias existentes para promover un mayor conocimiento del tejido empresarial fronterizo, mejorar su competitividad e impulsar la producción de productos y servicios comunes.

- Acción 2. Prospección de empresas en parques empresariales y promoción de productos y servicios. Conocimiento del tejido empresarial local para detectar oportunidades de mejora competitiva a nivel transfronterizo (encuesta a $1.000 \mathrm{em}$ presas/selección de 200).

- Acción 3. Aplicación de la prospección: transferencia de tecnología entre universidad y pequeña empresa. Lanzamiento de proyectos de colaboración innovadores de transferencia tecnológica para el desarrollo de la empresa y para que tenga acceso a mercados diferenciados y con un fuerte valor añadido. 20 diagnósticos.

- Acción 4. Creación de vínculos interempresariales. Definición y creación de productos y/o servicios que a través de la colaboración interempresarial transfronteriza mejoren la competitividad. 20 diagnósticos.

- Acción 5. Gestión y comunicación del proyecto, a través de un Comité técnico y Comité político ${ }^{12}$.

Una de las primeras actuaciones materializadas ha sido la elaboración de un Catálogo de Empresas Subcontratistas. El catálogo recoge información de empresas de Irun y Hondarribia subcontratistas que trabajan total o parcialmente para otras empresas-

11. Declaraciones realizadas en el marco de la presentación del proyecto Innov Mugabe, en Irun, en diciembre de 2010 y publicadas por el Diario Digital del Bidasoa, bajo el título "Primera reunión de balance de actividades del proyecto transfronterizo InnovMugabe liderado por Bidasoa activa"

12. Información recogida de la presentación del proyecto Innov Mugabe en su presentación pública realizada el 19-12-2010 y publicada en por el Diario Vasco bajo el título "El comité político de Innov Mugabe celebró su primera reunión" Consultar www.diariovasco.com 
clientes, fabricándoles partes o componentes de sus productos, y que quieren darse a conocer dentro y fuera de la comarca del Bidasoa. Otro tanto se ha realizado en los municipios que componen la Comarca Sud Pays Basque. En la página web www.bidasoasudpaysbasque.com figura ya el directorio que recoge a 6.000 de las 13.000 empresas ubicadas en los 14 municipios participantes. La plataforma web creada está vinculada directamente a las bases de datos de empresas de Bidasoa Activa y de la Communauté, por lo que cualquier cambio que se haga, se actualiza en ella inmediatamente. De esta manera se consigue que esa web sea una herramienta consolidada para el futuro, que no muera cuando termine el proyecto Innov Mugabe.

Una vez finalizado el primer catálogo, a finales del año 2011, la iniciativa transfronteriza comienza a dar nuevos pasos en la colaboración. Se trata, a partir de ese momento, de impulsar 40 proyectos piloto en dos categorías diferentes. En una, el objetivo es sacar al mercado productos y servicios Innovadores mediante la alianza de las empresas con los centros universitarios, que en la región son Mondragon Unibertsitatea (Irun) y la Escuela de Ingenieros Estia (Bidart). El plan incluye 10 propuestas con empresas del Bidasoa y otras tantas con empresas del Sud Pays Basque. Los otros 20 proyectos, también divididos en 10 por cada lado, tratan de fomentar los vínculos transfronterizos entre las propias empresas con el objetivo similar de crear nuevos productos y servicios que mejoren la competitividad.

Este plan se materializa en la iniciativa A3 Innov Mugabe, un programa cuyo objetivo es promover acciones de Innovación en el tejido empresarial de este territorio y cuyo concurso se resuelve a principios de año $2013^{13}$. 10 empresas del Bidasoa pueden acceder a los servicios de un centro tecnológico, un centro de formación o una ingeniería especializada, otras tantas en la Communauté. Entre las del Bidasoa cabe destacar que 7 de los proyectos seleccionados, serán realizados por centros especializados de la propia comarca. Se trata de los centros de formación IEFPS Bidasoa y La Salle, las ingenierías STB Ingenieros, Sisconsi y Prodising.

13. La resolución del programa "A3 Innov Mugabe" para el impulso de proyectos de innovación tecnológica se publica en marzo 2013. Las empresas beneficiarias y título de los proyectos son los siguientes BSM Informática: Diagnóstico de viabilidad para la adaptación de servicios online accesibles desde todo tipo de dispositivo, orientados al sector hotelero; PMT - Pulimentos Metálicos Txingudi: Análisis y Viabilidad técnica y económica para la fabricación de una nueva máquina para el sector aeronáutico; ALKAR, S.Coop: Análisis para el diseño y fabricación de multi-herramienta individual hidráulica a batería, con software controlador y certificado incorporado; POLIESTER GABIRIA: Diagnóstico Tecnológico para el diseño de un contenedor estándar porta-equipos (deportivos) para vehículos; ERREKALDE TXAKOLINA: Estudio para la Implantación de metodologías de "viticultura de precisión" mediante la obtención, análisis y aplicación de imágenes con microdron; SMAAP: Proyecto MAKAZAGA: Diseño y mecanizado de piezas especialmente innovadoras para prototipos de Ala Delta; IGORLE: Sincronización de un elemento rotativo con un elemento en desplazamiento lineal; OPPTIMIZA: Auditoría para la introducción de la tecnología móvil en entornos geográficos y su introducción a los productos de la empresa; ATELEI: Diseño, implementación e integración de Sistemas de Identificación por Radiofrecuencia en el ámbito de la seguridad industrial; BIGUMETRIK: Análisis de la oportunidad de creación de un laboratorio de metrología avanzada (www.bidasoa-sudpaysbasque.com). 
Todos los proyectos seleccionados tienen un objetivo final muy tecnológico, van desde el diagnóstico para la creación de un producto concreto o el análisis tecnológico necesario para la mejora de procesos de trabajo, hasta la creación de nuevas aplicaciones móviles. Más concretamente, encontramos proyectos relacionados con la creación de un laboratorio único en Gipuzkoa, estudios para la creación de nuevos productos de aeronáutica o deportivos, o diagnósticos de viabilidad para la adaptación de servicios online a todo tipo de dispositivos.

Pero, además de la relación iniciada en estos campos, se suceden iniciativas de colaboración y encuentros a lo largo del año 2012 y 2013 que tratan de poner en relación a empresas de uno u otro lado de la muga. Generalmente se inicia con la celebración de una jornada en la que se reúnen empresas de una determinada rama de actividad, a partir de la cual comienza a iniciarse un proceso de relaciones más o menos dirigido.

A mediados del año 2012 se reúnen en un centro de congresos de Irun 30 empresas del sector agroalimentario de ambos territorios, con el objetivo de identificar posibles proyectos de colaboración entre entidades de los dos lados de la frontera, en este caso para reflexionar sobre el sector agroalimentario a través de temáticas como nuevos productos o servicios, estrategia comercial y de marketing y recursos clave.

En otros encuentros se comparten también experiencias individuales con todo el grupo y a partir de éstas y de las relaciones que se establecen surgen iniciativas comunes. Como consecuencia, un stand con los productos del área ha estado presente en varias ferias gastronómicas: El Salón de Gourmets de Madrid, en "Gastronord" de Estocolmo y en la "Speciality and Fine Food Fair" de Londres. Además de los premios que a nivel individual obtienen determinados productores, se generan sinergias entre empresas del País Vasco, y ayudan a comercializar un territorio más allá de sus fronteras ${ }^{14}$.

Otro tanto se ha pretendido con el sector náutico, con la organización de un foro profesional transfronterizo. El pasado 12 de junio del 2012 se dieron cita en Hendaia profesionales del sector náutico en el primer foro transfronterizo organizado por la Communate Sud Pays Basque y Bidasoa Activa, en el marco del proyecto europeo Innov Mugabe. El objetivo del foro era desarrollar el sector de la Náutica, Deslizamiento y Actividades del Agua.

El mar y los espacios acuáticos a pie de montaña, son una imagen que otorga carácter propio a nuestro territorio, con $30 \mathrm{~km}$ de costas y 14 playas, el lago de Saint-Pée,

14. Las empresas cuyos productos han estado presentes en estas ferias son las siguientes: PARIES: elaboración de chocolates y otras dulces especialidades, ERROTA: aceites de nuez y avellana; HIRUZTA: txakoli; JEAN DE LUZ: conservería especializada en la transformación de pescados salvajes; TXOPINONDO: La elaboración de la sidra; LA BACALADERA: Variedad de productos que provienen de una misma fuente: el bacalao. Consultar más información en www.bidasoactiva.com 
la bahía de San Juan de luz/Ciboure, la bahía de Txingudi o los ríos Nivelle, Bidasoa y Untxin. En este territorio, el sector náutico representa múltiples actividades con un considerable potencial económico y un innegable atractivo turístico. El sector náutico cuenta con 120 empresas, más de 20 asociaciones con 3.000 socios y 4 puertos deportivos. El conjunto de estas actividades suma 4.500 empleos directos e indirectos.

El primer foro profesional invitó a representantes del sector náutico a reunirse en torno a cinco mesas de reflexión:

- Ordenación de los espacios y las instalaciones náuticas, en la que se dieron cita 18 empresas.

- Desarrollo de la oferta y apoyo a los proyectos, en la que se reunieron 16 empresas.

- Promoción y animación del sector, donde se citaron 22 empresas.

- Innovación, desarrollo sostenible y medio ambiente, a la que asistieron 16 empresas.

- Formación y empleo reunió a un total de 11 empresas.

Las personas responsables de la organización del Foro señalan que los objetivos del primer encuentro transfronterizo de actividades náuticas son, principalmente, el de favorecer los encuentros entre profesionales de ambos lados de la muga, aglutinar los actores del sector alrededor de una dinámica conjunta, favorecer el nacimiento de proyectos comunes y ser un referente para otros territorios transfronterizos ${ }^{15}$.

Éstas y otras iniciativas se están desarrollando al amparo del proyecto Innov Mugabe, la primera iniciativa de promoción empresarial que se desarrolla a ambos lados del Bidasoa. El proyecto, según sus responsables ${ }^{16}$, nace sin demasiadas expectativas. El Sur de Territorio de Lapurdi es un área que combina actividades de ocio y turismo con agricultura. Apenas hay tejido industrial y está casi en su integridad constituido por micropymes. Excepto alguna pequeña zona no hay concentración de empresas en un mismo lugar. La Comarca del Bidasoa, sin embargo, presenta un tejido industrial más consolidado, dominado por pymes pero con presencia de empresas de tamaño medio y con una ubicación física delimitada en polígonos industriales. Se hacía difícil prever cuales podían ser los campos que en el transcurrir de Innov Mugabe pudieran tener más desarrollo y se corría el riesgo de que cada zona materializara su trabajo de espaldas a la otra.

15. El encuentro es abordado de manera más amplia por Bidasoa Activa (2012) bajo el título "1er Foro Profesional Transfronterizo de actividades náuticas", recogido en su web; www.bidasoa-activa.com

16. Con el objeto de valorar los resultados del proyecto Innov Mugabe procedemos a entrevistar a los técnicos encargados de la materialización de este proyecto. En la misma abordamos cuestiones relativas al alcance real del proyecto, a las acciones iniciadas y a su continuidad, a la relación entre ambos socios y a las perspectivas de futuro que se plantean entorno a la colaboración entre Bidasoa Activa y la Communauté. 
Los resultados no han sido espectaculares, el número de empresas que ha participado en proyectos de colaboración entre uno y otro lado no es destacable pero las relaciones iniciadas han sido positivas y se han conseguido avances, al menos, en cinco áreas diferenciadas, tal y como apuntan los entrevistados.

En primer lugar, se han producido los primeros pasos en los que ellos denominan exportación, es decir, en la participación en eventos comunitarios donde se ha vendido destino y producto, a los que han acudido particulares en un stand común de toda la comarca. Se refieren a la participación en diferentes ferias en las que el producto del área se ha constituido en representante no sólo del Bidasoa sino de todo el País Vasco de un lado y otro de la frontera.

En segundo lugar, en el desarrollo del concepto Mugadian, o labrando y maquinando, es decir, impulsando proyectos de colaboración en toda la cadena de valor. En este caso las iniciativas se han desarrollado en el sector agroalimentario realizando jornadas de puertas abiertas, seminarios, folletos..., que han impulsado contactos personales que se están materializando en proyectos y productos que surgen de esta colaboración.

En tercer y cuarto lugar, en la realización de planes estratégicos y jornadas específicas para determinados subsectores, el náutico por un lado y el sector de las artes gráficas por otro. Aunque en ambos casos la demanda ha venido del área francesa, el esfuerzo por incorporar empresas del Bidasoa ha sido importante, especialmente en el caso náutico.

Finalmente, en la materialización de preproyectos, con un total de once convenios de colaboración entre empresas de un lado y otro de la frontera, además de las agroalimentarias, en campos tan diversos como la relación entre empresas de muebles y de señalítica, ingeniería con panadería, náutica con material deportivo, etc.

Sin ser un proyecto pretencioso, Innov Mugabe ha propiciado un acercamiento entre los territorios fronterizos que está dando pie a la participación en proyectos de menor entidad, como Euskadi - Aquitania, donde se ha subvencionado la elaboración de un catálogo de industrias agroalimentarias de ambas zonas.

Innov Mugabe ha sido la primera acción conjunta en materia de desarrollo económico y los resultados han sido valorados positivamente por unos y otros. Como consecuencia, ya se ha presentado otro proyecto desde Bidasoa Activa y la Communauté, en colaboración con los centros tecnológicos que operan en el área, como la Universidad de Mondragón y la escuela de Ingeniería Estia. El proyecto de Responsabilidad Social Territorial Transfronteriza (RESOT) tiene por objeto la transformación de la zona hacia un espacio de Responsabilidad Territorial en materia económica, social y medioambiental. Al igual que el anterior, está enmarcado en el Programa de Cooperación Territorial Transfronteriza (POCTEFA) para reforzar la integración económica y social de la zona fronte- 
riza España-Francia-Andorra mediante el desarrollo de actividades transfronterizas a través de estrategias conjuntas a favor del desarrollo territorial sostenible. El proyecto viene a dar continuidad a la relación que Innov Mugabe había promovido ${ }^{17}$.

Es más, según apuntan los responsables del proyecto en las entrevistas, la colaboración en materia de desarrollo económico va a continuar, independientemente de que cuente o no con ayuda económica puesto que las entidades responsables han puesto las bases para que las herramientas que se han realizado sean renovadas continuamente por los propios usuarios (catálogo de empresas, GIS...) y se han comprometido a mantener una dinámica de colaboración continuada.

De hecho, si antes de la crisis el trasvase de población de un lado a otro de la muga era evidente, ahora son las empresas del Bidasoa y de toda Gipuzkoa las que buscan ampliar su mercado en el País Vasco Francés. Formar, asesorar y dirigir este interés es competencia de Bidasoa Activa y de la Communauté Sud Pays Basque, y por ello está colaboración es cada vez más demandada.

Pero en este desarrollo hay una entidad que pierde peso, el Consorcio Transfronterizo. La Communauté ha adquirido en Marzo de 2013 la subcategoría de Aglomeración, consecuencia del incremento de población, preferentemente de Hendaia, y que se traduce en la asunción de nuevas competencias, especialmente infraestructuras. Con ello, se aleja aún más la posibilidad de que la conurbación del Bidasoa adquiera peso administrativo mientras las relaciones entre entidades supramunicipales se incrementa.

\section{De la Conurbación del Bidasoa a la Comarca Bidasoa-Sud Pays Basque. A modo de conclusión}

En un momento en el que la Conurbación del Bidasoa ha pasado de una unidad física a una relación dinámica con población que se mueve de un lado a otro por cuestiones de residencia o de trabajo, la entidad que surge para impulsar esta unión, el Consorcio, no se está desarrollando a una velocidad similar. Continúa promoviendo actuaciones en áreas similares a las de hace década y media y a pesar de los avances conseguidos, no ha abordado la diversificación de áreas para las que en un principio fue creado, como la promoción económica y la asunción de competencias dotacionales.

Es más, la crisis económica, una oportunidad para asesorar y dirigir al flujo empresarial interesado en la búsqueda de nuevos mercados al otro lado de la ría, está siendo

17. Aunque todavía en fase de proyecto RESOT ha comenzado su andadura. La información que va generando se puede consultar en su página web www.resot.eu 
un lastre para el Consorcio Transfronterizo, que ha visto como su presupuesto se reducía a cifras críticas consecuencia de los recortes de las entidades municipales.

Además, el desarrollo que la Communauté Sud Pays Basque ha ido adquiriendo desde su creación el año 2005 ha llevado a que Hendaia deje en manos de ella algunas de las competencias que inicialmente había previsto que fueran abordadas de manera conjunta desde la Conurbación. A su vez, la Communauté ha asumido la responsabilidad de algunos de los campos que el Consorcio tradicionalmente había trabajado, como cultura o turismo. Las relaciones se mantienen pero Hendaia mira ahora también a los $11 \mathrm{mu}-$ nicipios que componen su comarca.

Todo indica que avanzamos en las relaciones transfronterizas del Bidasoa de la Conurbación de tres municipios al entendimiento entre Bidasoa Activa y la Communauté Sud Pays Basque. Es más, a propuesta del Consorcio, el entente Bidasoa Activa-Sud Pays Basque está preparando un nuevo proyecto consistente en ofertar un bono turístico para visitar los museos ambos lados de la ría. Los proyectos continúan, los técnicos son los mismos pero el ámbito de actuación se amplía.

La nueva área geográfica permite abordar actuaciones en campos que en las dos décadas de relación entre un lado y otro del Bidasoa no se habían materializado. El proyecto Innov Mugabe abre una puerta a la colaboración entre empresas y entre centros tecnológicos, una relación que no ha hecho más que empezar y que tiene visos de continuar, como lo demuestra la prórroga reciente que ha tenido este proyecto. El interés mostrado por el empresario parece augurar un interesante campo de desarrollo tanto por la afluencia masiva que han tenido las jornadas realizadas y los cursos que desde Bidasoa Activa se han orientado a enseñar sobre cómo trabajar en Francia.

Las relaciones culturales, turísticas y empresariales tienen ahora un nuevo marco de desarrollo en el Bajo Bidasoa-Sud Pays Basque e incluso, quien sabe, si en el futuro también las infraestructuras básicas y dotacionales puesto que la Comunauté ya ha emprendido proyectos transfronterizos en estas áreas con otros municipios del Bidasoa, en territorio de Navarra. Tal vez ha llegado el momento de que los valles navarros del Bidasoa (Baztan, Malaerrreka y Bortziri), limítrofes con la Comarca Sud Pays Basque y Bajo Bidasoa, se planteen unas relaciones y un futuro en común. 


\section{Bibliografía}

Adebisa-Bidasoa Activa (1993) Informe anual. Publicación anual Irún.

Bidasoa Activa (2012) $1^{\text {er }}$ Foro Profesional Transfronterizo de actividades náuticas, www.bidasoa-activa.com. Consulta 30-04-2012.

Agglomeration sud pays basque (2013) "20052012. D'une Communauté de Comunes à une Agglomération". www.agglo-sudpaysbasque.fr. Consulta 30-4-2013.

Goizane Álvarez (2010) Primera reunión de balance de actividades del proyecto transfronterizo Innov Mugabe liderado por Bidasoa activa. Diario Digital de la Comarca del Bidasoa. Irun. www.bidasoaldia.com. Consulta 21-3-2013.

Ibarra P. e Ikardo I. (1997) Movimientos sociales transfronterizos en la bahía de Txingudi. La construcción del espacio vasco-aquitano. Servicio Editorial de la Universidad del País Vasco. Lejona: 115-128.

Llusà Torra R. (2001) Desarrollo local en el espacio-red. El área urbana de Manresa como sistema territorial local. Actas del XVII Congreso de Geógrafos Españoles. Oviedo. 378-381.

Morondo I. (2010) El comité político de Innov Mugabe celebró su primera reunión. Diario
Digital de Gipuzkoa. Donostia. www.diariovasco.com (Consulta 2-5-2013).

Plaza B. y Velasco R. (1999) Agencias de desarrollo y territorio: La experiencia del País Vasco. Ciudad y Territorio. Estudios Territoriales, XXXI(122). 835-853.

Resot (2013) Responsabilidad Social Transfronteriza. www.resot.es

Rico del Amo, R. (1994) Presentación. Informe Anual. Memoria de Actividades Adebisa-Bidasoa Activa 1993. Edificio Kostorbe. Irun.

Sallaberry J.B. (2012) Lo transfronterizo no ha ido tan rápido como debía y ahora lo estamos pagando. En Morondo (corresponsal) Diario Digital de Gipuzkoa. Donostia. www.diariovasco.com (Consulta 2-5-2013).

Sallaberry J.B. (2012) Vamos a frenar el crecimiento salvaje, pero no la llegada de españoles. En Ezquiaga (corresponsal) Diario Digital de Gipuzkoa. Donostia. www.diariovasco.com (Consulta 2-5-2013).

Sese Sarasti E. (1994) La planificación estratégica en las experiencias de desarrollo local. Herramientas : revista de formación para el empleo, 3(33). 18-21. 